\title{
The Management of Leprous Rhinitis
}

\author{
R. P. E. BARTON \\ Ear, Nose and Throat Department, St Mary's Hospital, London W.2
}

\begin{abstract}
The management of leprous rhinitis is discussed. In patients with early changes in the nose it is thought that local treatment is of psychological rather than of any great physical value. There is, however, a group of patients with early lepromatous leprosy whose nasal involvement is severe and out of all proportion to their general clinical state. It is possible that intensive local treatment of the nose in these patients may help to prevent deformity. Patients with advanced nasal changes giving rise to atrophic rhinitis and external deformities gain much physical, as well as psychological, relief from regular local care of the nose, and this is described below.
\end{abstract}

\section{Introduction}

It is well known that the nose is commonly involved in lepromatous leprosy, and it has been shown recently (Barton et al., 1973) that this involvement occurs early in the disease process. Furthermore, the changes that are seen; both clinically and histologically, are frequently more severe than might be expected from the systemic state of the patient. The detailed description of these changes is presented elsewhere, but for the purpose of this paper they may be summarized as follows.

In early lepromatous leprosy the nose is involved in an inflammatory, granulomatous process which initially causes obstruction of the nasal airways, increased discharge of mucus or muco-purulent matter, occasional bleeding and, under dry atmospheric conditions, crust formation.

If this process is allowed to continue without treatment it will eventually lead to destruction of the normal anatomical and physiological state of the nose, with these consequences: (a) Internal changes. The classic picture of atrophic rhinitis appears (Reynaud and Languillon, 1961) with erosion of the lateral nasal walls, septal perforation, ozaena, cacosmia and hyposmia (Barton, 1973). (b) External deformity. The loss of the supporting nasal septum, particularly superiorly and anteriorly (the columella), and also of the anterior nasal spine leads to the typical deformity seen in advanced lepromatous leprosy.

\section{The Importance of Treatment}

It would be all too easy to dismiss these changes as an unimportant aspect of leprosy work. However, while working on other projects at Victoria Hospital, Dichpalli, in central southern India, the writer was fortunate enough to be able not only to treat the nasal lesions in leprosy patients but also to see just how important the patients themselves considered their nasal symptoms to be. It became very obvious that local treatment of the nose was a most important and valuable part of the overall care of these patients. 
Lest it should be thought that nasal care is too time-consuming or too specialized a subject for the leprologist to tackle without training in the methods of the ear, nose and throat surgeon, it is worth pointing out that, with a little practice, it should be possible to treat between 35 and 40 patients per hour.

\section{Rationale of Treatment}

Patients whose nose was still in the early stage of involvement often had quite severe symptoms. They were able, in the hospital situation. to observe and talk to others with more advanced illness who had already developed nasal deformity and this, quite naturally, caused concern that they too would suffer the same fate. While the patients of ten realized that the medicine which they received by mouth (normally dapsone) was in fact doing them good, it became clear on talking to them that they found it hard to relate the state of their nose to the tablets that they were getting. Therefore, although the clinical state of the nose and the bacilli present in the nasal mucosa show a favourable response to dapsone therapy within a matter of weeks (Barton et al., 1973), it was of ten difficult to convince these patien ts that their noses would get better without any local treatment.

Patients with more advanced changes present a different problem: basically their nasal treatment is that of atrophic rhinitis. Much has been written in the E.N.T. literature on this subject and it may be briefly summarized as follows.

\section{Local Treatment}

Removal of crusts, either mechanically or by irrigation with warm isotonic solutions, is followed by painting or spraying of the nasal cavities with some suitable medicament. Many substances have been used, but a $25 \%$ solution of glucose in glycerine has been, perhaps, the most widely favoured. This is said to prevent the adherence of fresh crusts and to inhibit colonization of the nose by saprophytic organisms. Mukarji (1973), in a review of some of the earlier leprosy literature, notes several references to local treatment of the nose. A wide variety of preparations are mentioned, these including various borax solutions, iodized glycerine, $5 \%$ aqueous chromic acid, and iodized hydnocarpus oil.

\section{Medical Treatment}

Many authors have postulated a hormonal factor in the causation of atrophic rhinitis. Certainly it is more common in females and exacerbations are often associated with puberty and pregnancy. Oestrogens have been of value in treating this condition, although Taylor and Young (1961) suggested that their efficacy may be due to their vasodilator effect on the nasal mucosa rather than to any systemic hormonal action. Other vasodilators, such as nicotinic acid. and drugs intended to increase the nasal secretions, such as potassium iodide, have also been tried.

Many bacteria have been isolated from the noses of patients with atrophic rhinitis, but there is little evidence that any specific organism causes the disease (Foxen, 1971). For this reason it is not surprising that antibiotics are of limited use, even when the sensitivity of any bacteria in the nose can be determined. Generally speaking, however, it is fair to say that the present medical treatment of atrophic rhinitis is disappointing and that further work is needed to discover more efficacious drugs. 


\section{Surgical Treatment}

Young (1967) recommended closure of the nostrils for periods of up to 4 years, and stated that when the nose was re-opened the mucosa was seen to have a normal appearance. Previously he had found that partial closure or narrowing of the nose gave palliation in some cases but was not actually curative. Narrowing of the nasal cavities to help atrophic rhinitis was tried as long ago as 1916 by Lautenschlager. He, using a trans-antral approach, mobilized the nasal wall of the maxillary antra medially in order to decrease the size of the nasal cavities. Wittmaach modified this operation to include re-routing of the parotid salivary duct into the antrum-unfortunately this manoeuvre resulted in salivation from the nose at mealtimes!

Autografts of bone or cartilage placed submucosally have the disadvantage of undergoing resorption, while foreign material runs the risk of extrusion and rejection. This risk has been reduced since the introduction of relatively inert substances such as teflon and Silastic. Attempts to improve the blood supply to the nasal mucosa by stellate ganglion block (Sharma and Sardana 1966) require repeated injections into the neck. If stellate ganglion block is successful, then logically cervical sympathectomy should be considered.

Recently, Ssalli (1973) has recommended amputation of the middle turbinates, and claims good results from this procedure (91\% of a small series were said to have improved). However, in many cases of atrophic rhinitis resulting from advanced lepromatous leprosy, personally observed, it was striking how often the upper part of the nasal cavities, at the level of the middle turbinates and above, appeared to be normal, even when gross atrophic changes were present lower in the nose. Middle turbinectomy can only serve to increase the already pathologically enlarged nasal cavities of atrophic rhinitis. As such it is difficult to see why this line of treatment should be effective.

Mention having been made both of patients with early changes in the nose and the very large group with atrophic rhinitis, it is worth considering briefly a small but extremely interesting group. These are the patients who, early in their illness or when their leprosy is rapidly "down-grading", suffer a very acute nasal infection which, together with their general systemic state, continues to deteriorate temporarily after the institution of dapsone or other anti-lepromatous chemotherapy. In this group it is quite probable that careful local treatment of the nose helps to prevent those changes that lead to nasal deformity, in that the inflammation may be controlled until such time as the systemic chemotherapy begins to reverse the intranasal pathology. If treatment is begun early enough, deformity can undoubtedly be prevented or arrested.

\section{Details of Treatment}

Various methods were experimented with, but in the time available for developing an efficient and effective treatment plan it was not possible to undertake adequately controlled trials of the different methods used. Therefore, the following scheme was arrived at as a result of favourable subjective impressions gained by both patients and doctor.

\section{(A) EARLY CASES}

It has already been observed that it is the systemic therapy that is of prime importance-no lepromatous nose will be cured by local treatment alone. 
However, careful local treatment will never harm the nose and therefore. as the psychological factor is of such importance in the overall management of these patients, anyone with nasal symptoms, provided there was some intranasal pathology, was accepted for regular care.

What was considered to be of great importance was to instruct the patients firmly that they must not attempt to "pick" or in any way traumatize their nose. In a consecutive series, personally observed, of patients with all forms of leprosy. over half ( 78 out of 150: or 52\%) had some degree of sensory loss in the nose. This is probably an important aetiological factor with regard to ulceration of the mucosa over the nasal septum and thence in perforation of the septum and eventual external deformity.

\section{(B) LATE CASES-(including those with rapidly advancing infection)}

Surgical intervention along any of the lines previously mentioned was not possible at the time of this investigation. Similarly the various forms of systemic medical treatment recommended for non-lepromatous atrophic rhinitis-the results of which are in any case frecuently disappointing-was not considered worth exploring in the time available. The treatment employed was, therefore, entirely local in nature, and the procedure eventually chosen is outlined below.

(1) Removal of crusts. This was considered to be the most important single part of treatment. Removal of crusts must be both careful and meticulous, as any small pieces remaining act as a nidus for further crust formation. Any trauma to the delicate nasal mucosa will aggravate the local condition and could increase the risk of nasal deformity. Two hands are needed for removing crusts-one to hold a nasal speculum in order to dilate the nostrils, and the other to hold a pair of nasal dressing forceps (Tilley's or Heath's patterns are suitable). It is, therefore, clear that a good independent light source is required. and the author favours a convex head-mirror used to reflect light from a "bull's eye" or other strong lamp placed behind the patient. Alternatively, a battery- or mains-operated headlamp may be used; a torch held by an assistant was found to be not satisfactory for fully illuminating the whole of the nasal cavities. When hard adherent crusts were encountered patients were shown how to irrigate the nose so as to soften and loosen the crusts before mechanical removal was attempted. An effective solution was found to be equal parts, by weight, of sodium bicarbonate, sodium borate and sodium chloride, $15 \mathrm{~g}$ to be dissolved in $500 \mathrm{ml}$ of warm water.

(2) Local medicament. Immediately after removal of all the crusts, the nose was carefully painted with a liberal amount of an ointment composed of:

$\begin{array}{lr}\text { Vaseline } & 1 \mathrm{~kg} \\ \text { Glycerine } & 200 \mathrm{~g} \\ \text { Vioform } & 300 \mathrm{~g} \\ \text { Crystal violet } & 5 \mathrm{~g}\end{array}$

This ointment was applied to all accessible parts of the nasal cavities on cotton-wool-tipped sticks, a plentiful supply of which is needed. Several different ointments were tried and discarded for various reasons, before this particular one was chosen. A preparation of $25 \%$ glucose in glycerine, contrary to what is generally accepted, resulted in several cases in the colonization of the patient's nasal cavities by a dry, white fungal growth. It was not possible to identify this growth further and, while it did not appear to cause any local symptoms or distress, the use of glucose in glycerine was abandoned. 
Various types of oily nasal drops were of some value, but it was found that they tended to dry rather rapidly and they were not employed in routine in-patient care. Supplies were gratefully accepted by many of the hospital's out-patients, but the efficacy of their action was not fully assessed. A simple paste of Vaseline and Vioform was originally used. but the disadvantage of this preparation was that it tended to dry out and so became more difficult to apply to the nasal mucosa as it seemed to lose its "stickiness". This problem was overcome by adding glycerine: the ointment then became easy to apply to the mucosa and became pleasant to handle in all respects.

Vaseline, which was used elsewhere in the hospital in the care of hands and feet, helped to keep the crusts, which in many patients reformed very quickly, soft and easy to remove. Vioform (iodochlorhydroxyquinolone) is an antiseptic that is bactericidal and also active against many fungi: it does not cause sensitivity reactions, nor do organisms become resistant to it. It has the added advantage of being cheap and easily available in the form of Enterovioform tablets which may be crushed and incorporated in the ointment. (Each Enterovioform tablet contains $250 \mathrm{mg}$ of the active principle.) The ointment was used in this form (i.e. Vaseline, Vioform and glycerine) for some time before it was decided to add crystal violet. This also has antiseptic properties and has the additional advantage of giving the ointment an impressive deep blue colour. Apart from its psychological value (the patients were unanimously in favour of the new colour when this was added) it had the additional advantage of acting as a marker. and thus enabling one to see how long the ointment remained present. and therefore presumably effective, in the noses of different patients.

(3) Frequency of treatment. Initially treatment was given on a daily basis, but many patients failed to attend regularly: also the crystal violet which remained in the nose showed that in many patients traces of ointment could be detected even after five to seven days. Eventually the Nose Clinic at Victoria Hospital functioned on Mondays, Wednesdays and Fridays. Patients were given cards to be signed at each attendance, and this improved both the regularity of attendance and the results obtained.

It was most gratifying to see the improvement in the noses of our patients: it was sometimes dramatic, and frequently very good. A few patients did badly despite what was considered to be adequate local and systemic treatment, and the reasons for this are not altogether clear. Though there was not time for full investigation, it is possible that those whose nasal condition deteriorated were patients infected with bacilli resistant to dapsone.

\section{Conclusions}

It is hoped that these observations and suggestions on the treatment of the nasal lesions in leprosy will stimulate those who have, or can develop, the facilities to use them as widely as possible. While careful removal of crusts is of great importance, there is still much scope for the further development of local preparations to be used intranasally. Initially it was thought that an anti-lepromatous drug could be incorporated in to the ointment used, but in patients with late nasal lesions the degree of infectivity is not high. In patients with early lepromatous leprosy it is the oral drugs that are important in producing both general and nasal improvement. However, in the group of patients already mentioned with very rapidly advancing nasal infection. it is possible that the 
intranasal use of anti-lepromatous drugs may prove to be of válue. Certainly further work is needed.

In the present study no surgical treatment was attempted in the time available. and it is doubtful if Young's operation of total closure of the nostrils would be tolerated by leprosy patients. It is possible that the likely palliation resulting from the insertion of teflon implants under the mucosa of the lateral walls and floor of the nasal cavities would be worthwhile and this method could profitably be explored.

\section{Acknowledgements}

My grateful thanks are due to Dr Frank Davey for his initial encouragement and help in this work; to Dr L. M. Hogerzeil and staff at Victoria Hospital, Dichpalli, for granting all facilities. and especially to Mr B. Deena Dayalu whose assistance was invaluable. My thanks are also due to the Board of Governors of St Mary's Hospital, London, for allowing me leave of absence, and to the British Leprosy Relief Association (LEPRA) for their generous support.

\section{References}

Barton, R. P. F. (1974). J. Laryngol. ()tol. In press.

Barton, R. P. E., Davey, T. F., McDougall, A. C., Rees, R. J. W. and Weddell, A. G. M. (1973). Proceedings of Tenth International Leprosy Congress, Bergen.

Foxen, E. H. Miles (1971). In Diseases of the Ear, Nose and Throat, Vol. 3 (Ed. Ballantyne and Groves). Butterworths: London.

Mukarji, D. (1973). Personal communication.

Reynaud, J. and Languillon, J. (1961). Ann. Oto-Laryngol. 78, 44.

Ssali, C. L. (1973). J. Laryngol. Otol. 87. 397.

Sharma, A. N. and Sardana, D. S. (1966). J. Laryngol. Otol. 80, 184.

Taylor, M. and Young, A. (1961). J. Laryngol. Otol. 75, 574

Young, A. (1967). J. Laryngol. Otol. 81, 515. 